University of Nebraska - Lincoln

DigitalCommons@University of Nebraska - Lincoln

John R. Hardy Papers

Research Papers in Physics and Astronomy

5-15-1971

\title{
Calculation of Point-Defect Energies and Displacements in Alkali Halides Using the Lattice-Statics Method
}

Arnold Karo

University of California, Livermore, California

John Hardy

University of Nebraska - Lincoln

Follow this and additional works at: https://digitalcommons.unl.edu/physicshardy

Part of the Physics Commons

Karo, Arnold and Hardy, John, "Calculation of Point-Defect Energies and Displacements in Alkali Halides Using the Lattice-Statics Method" (1971). John R. Hardy Papers. 21.

https://digitalcommons.unl.edu/physicshardy/21

This Article is brought to you for free and open access by the Research Papers in Physics and Astronomy at DigitalCommons@University of Nebraska - Lincoln. It has been accepted for inclusion in John R. Hardy Papers by an authorized administrator of DigitalCommons@University of Nebraska - Lincoln. 


\title{
Calculation of Point-Defect Energies and Displacements in Alkali Halides Using the Lattice-Statics Method*
}

\author{
Arnold M. Karo \\ Lawrence Radiation Laboratory, University of California, Livermore, California 94550 \\ and \\ John R. Hardy \\ Behlen Laboratory of Physics, University of Nebraska, Lincoln, Nebraska 68508 \\ (Received 27 March 1970)
}

\begin{abstract}
The method of lattice statics, in the zero-order approximation, is used to evaluate the Schottky-pair formation energies and the displacement fields about positive- and negative-ion vacancies for all alkali halide crystals with rock-salt structures. The deformation-dipole model, used extensively in earlier work on the lattice dynamics of these crystals, is employed to describe the host lattice. Long-range Coulomb interactions are included explicitly, and short-range repulsive interactions are described by a simple Born-Mayer potential. Comparison is made with earlier Mott-Littleton-type calculations and very satisfactory agreement is found. Comparison with available experimental values is somewhat less satisfactory, particularly regarding the lithium salts; but for "typical" alkali halides, e.g., $\mathrm{NaCl}$ and $\mathrm{KCl}$, the calculated values are within $10 \%$ of the experimental values.
\end{abstract}

\section{INTRODUCTION}

In 1938, Mott and Littleton ${ }^{1}$ presented their classic calculation of the formation energies of Schottky pairs in ionic crystals. The Schottky pair is defined as a pair of positive- and negative-ion vacancies, and the formation energy is that energy required to remove the ions from the vacated lattice sites and place them on the crystal surface.

The Mott-Littleton and subsequent calculations have been based on the assumption that the crystal energy can be written as a sum of pairwise terms. In the simplest case, the lattice energy is considered to be composed of the Coulomb energy of the positive and negative ions and the energy associated with a short-range repulsive interaction between nearest neighbors. The latter can be said to arise from overlap of the electron densities of neighbor ing ions as these are brought together in the crystal. The increase in local electron density with decreasing internuclear separation results in increasing Coulomb and exchange contributions that stabilize the lattice. Calculations by Löwdin ${ }^{2}$ support such a simple model although indicating the need for considering three- and possibly four-body terms in lattice-energy calculations.

As Mott and Littleton have shown, the energy required to create a Schottky pair is not simply that needed to remove the ions from their lattice sites and place them on the surface. If this were the case, the formation energy would be the lattice energy per ion pair. However, the lattice energy per pair is typically about $8 \mathrm{eV}$, whereas observed formation energies are about $2 \mathrm{eV}$. The difference arises because the lattice about a defect does not remain undistorted and unpolarized. One thus infers that the relaxation energy, i. e., the energy recovered when the lattice polarizes and distorts, is of the order of $6 \mathrm{eV}$.

In any theoretical computation, the formation energy appears as the difference between two larger quantities - the lattice energy and the relaxation energy -and care in evaluating these quantities is therefore important. Since the lattice energy can be precisely computed without difficulty, it is the relaxation energy that presents the problem, and the Mott-Littleton technique has been used to date to calculate this value. Such defect calculations have been characterized as "semidiscrete" 3 : In such calculations the lattice is divided into two regions labeled I and II. The first region, I, is treated on a discrete atomistic basis, while the second region, II, is represented by a continuum approximation. In simple Mott-Littleton calculations region I includes only the defect and its nearest neighbors and the second region is considered as a polarized dielectric continuum. Recent calculations by Scholz ${ }^{4}$ have extended region I to include more than 200 ions, region $I I$ again being represented by a dielectric continuum. In all these calculations there is the difficulty of matching regions I and II at the boundaries. For defects in metals it has been found ${ }^{3}$ that Mott-Littleton calculations would require region I to be inordinately large for the boundaries to be in the true continuum region. For ionic crystals, if the defect is charged, there is the additional complication of allowing for the long-range forces exerted by the defect on the ions of the host lattice. These forces cause the ions to displace and polarize, creating additional problems 
in the matching of regions I and II.

In an earlier paper ${ }^{5}$ the formalism for the method of lattice statics was presented, and it was shown that the formation energies of charged point defects in ionic crystals could be calculated without the artificial dichotomy between regions $I$ and $\Pi$. In that paper the principal concern was the method of calculating the displacements and polarizations and establishing their asymptotic behavior far from the defect. Our purpose in this paper is to present the results of numerical calculations for the rock-saltstructure alkali halide sequence based on the latticestatics method and with assumptions about ionic interactions similar to those made by Mott and Littleton. We shall restrict the calculations to the formation energies of Schottky pairs; however, the proposed method is general and can be applied equally well to other point defects such as substitutional impurities and interstitials.

\section{THEORY}

In this paper, we shall not repeat in detail the formal theory given in full in Ref. 5. It is worth emphasizing, however, that the crucial step in all these calculations is the Fourier transformation of the equilibrium equations for displacements and polarizations of the ions. It should be noted also that the ionic dipole moments must appear as essential additional degrees of freedom having their own equilibrium equation since, for a charged defect, a generalized force is applied to these electronic dipole moments as well as to the displacements. The model used here to describe the lattice is the deformation-dipole model also discussed in detail in our earlier paper. ${ }^{6}$. This model is a generalization of ideas originally developed by Szigeti ${ }^{7}$ for treating long-wavelength lattice vibrations or periodic distortions of arbitrary wavelength. Because of the finite number of ions in a lattice, it follows that there are but a finite number of allowed distinct wave vectors. These vectors are such that the number of Fourier components of the displacements, or of the dipole moments, is exactly equal to the total number of degrees of freedom of the lattice as a whole. From the detailed derivation of the lattice-statics method in Ref. 5, we can extract the following basic equations [i.e., the definition of the energy function $W$ and Eqs. (2.16) and (2.17) of Ref. 5] on which the present calculations are based :

$$
\begin{aligned}
W= & X(I: \vec{\xi}, \vec{\mu})+Y(\vec{\xi}, \vec{\mu}), \\
Y_{\min }= & \frac{1}{2}\left[\tilde{E}(\tilde{S} U+1) U^{-1} \vec{\xi}_{\min }+\tilde{V} \vec{\xi}_{\min }+\tilde{E} \vec{\mu}_{\min }\right], \\
Y_{\min }= & \frac{1}{2}\left\{\left[\tilde{V}+\tilde{E} C^{-1}(1+\tilde{S} U) U^{-1}\right] M^{-1}\right. \\
& \left.\times\left[U^{-1}(1+U S) \tilde{C}^{-1} E+V\right]\right\}+\frac{1}{2} \tilde{E} C^{-1} \alpha E,
\end{aligned}
$$

in which $\vec{\xi}$ and $\vec{\mu}$ denote the displacements and mo- ments in the region where the potential-energy function is harmonic. In the first equation, the total energy of the solid is expressed as the sum of two terms. The first term represents the interaction between the defect and the ions of the host lattice, and the second term represents the energy stored in the distorted and polarized but otherwise perfect lattice. The distortion is assumed to be within the limits of the harmonic approximation. The total energy $W$ of the solid is minimized with respect to both the displacements and the dipole moments, and both quantities are then eliminated by expressing them in terms of the force exerted by the defect. As a result we have Eq. (2), from which Eq. (3) follows by substituting the appropriate expressions for $\vec{\xi}_{\mathrm{min}}$ and $\vec{\mu}_{\mathrm{min}}$. The matrices have been defined in Ref. 5, and the quantity $Y_{\mathrm{min}}$ in Eq. (3) is the energy stored in the distorted perfect lattice. If the first term in Eq. (3) is expanded as a power series in the nuclear displacements and dipole moments, it can be show $n^{5}$ that the change in energy of the crystal when the lattice is allowed to relax and polarize (i. e., the relaxation energy $E_{R}$ ) is, to first order in $\vec{\xi}_{\text {min }}$ and $\vec{\mu}_{\text {min }}$, given by

$$
E_{R}=-Y_{\min } \text {. }
$$

The expression for $Y_{\mathrm{min}}$ is written entirely in terms of the forces, both Coulomb and repulsive, exerted by the defect on the lattice ions at their unrelaxed positions. We refer to this as the zero-order approximation, and the calculations in the present paper are made within this approximation.

The evaluation of $E_{R}$ is accomplished by Fourier transforming Eq. (3) and obtaining a sum over the allowed wave vectors, given in the following form:

$$
\begin{aligned}
E_{R}= & -\frac{1}{2} \sum_{\vec{q}}\left[\tilde{V}+\tilde{E} C^{-1}(1+\tilde{S} U) U^{-1}\right] M^{-1} \\
& \times\left[U^{-1}(1+U S) \tilde{C}^{-1} E+V\right]-\frac{1}{2} \sum_{\mathbb{q}} \tilde{E} C^{-1} \alpha E,
\end{aligned}
$$

in which the direct-space vectors and matrices are replaced by their Fourier transforms. Two important points should be emphasized. First, at no stage in this calculation has a distinction been made between region I and region II; the expression for $Y_{\mathrm{min}}$ refers to the lattice as a whole. Second, the long-range Coulomb forces, after Fourier transformation, can be handled by the Ewald $\theta$-function transformation ${ }^{8}$ in the manner described in Appendix I of Ref. 5. The zero-order approximation is particularly convenient because the actual displacements and dipole moments need not be introduced or evaluated in treating these long-range forces. To proceed beyond this approximation, techniques must be followed similar to those used in treating metals by the lattice-statics method, ${ }^{3}$ where the displacements of close neighbors of the defect were explicitly considered-these being the largest and also possibly outside the limits of the harmonic approximation. 


\section{RESULTS AND DISCUSSION}

It is relevant to summarize results of a survey of the alkali halide Schottky-pair formation energies and associated displacement fields about the vacancies. The energy calculations have been carried out within the zero-order approximation, and the results can be compared appropriately with MottLittleton-type calculations and with experimental data. In Table I we collect the formation energies of Schottky pairs evaluated in this way. Listed separately are the energies required to remove the positive and negative ions to infinity $\left(E_{+}\right.$and $\left.E_{-}\right)$, the lattice energy per unit cell $\left(E_{L}\right)$, and the formation energy of the Schottky pair $\left(E_{S}=E_{+}+E_{-}+E_{L}\right)$. Implied is an assumption that the energy of the perfect crystal can be written as a sum of pairwise terms. Thus, for the lattice energies we have a simple Born-Mayer form

$$
E_{L}=-e^{2} \alpha / r+6 A e^{-r / \rho}
$$

restricting the short-range interaction to nearestneighbor ions. The constants $A$ and $\rho$ are determined in the usual way from the lattice-equilibrium condition and the observed compressibility. Taken together, these define the first and second derivatives of the short-range interaction and thereby $A$ and $\rho$. The matrix $M$ is essentially the dynamical matrix, without pre- and postmultiplication by the diagonal mass matrix, defined for the deformationdipole model. ${ }^{6}$

Tables II and III summarize our calculations of the displacements of neighboring ions about the defects and along several symmetry directions for each alkali halide. Only representative results are given for the symmetry directions in Table III. Complete data are available upon request. ${ }^{9}$ The calculations follow from the expression

$$
M \vec{\xi}=U^{-1}(1+U S) \tilde{C}^{-1} E+V,
$$

or, by the Fourier transform

$$
M Q=U^{-1}(1+U S) \tilde{C}^{-1} E+V,
$$

which are Eqs. (2.14) and (3.1) of Ref. 5. One then back-transforms,i. e., sums the Fourier series for the direct-space displacement of each ion. We have, in fact, been somewhat more sophisticated in calculating the displacements versus the relaxation energy, since the short-range force $V$ is evaluated at the first-neighbor relaxed position. In doing this, we note that evaluation of the firstneighbor displacements $\vec{\xi}_{N N}$ about the defect in volves Eq. (7), which is nonlinear in these displacements since the short-range force is itself a function of the first-neighbor displacement. The evaluation can be done iteratively and, once accomplished, yields the appropriate form of $V$ for use in calculating the relaxation energy of any neighbor. Stated more precisely, we know the ap- 
TABLE II. Displacement components for certain near neighbors about a positive-ion vacancy $(+)$ or negative-ion vacancy $(-)$. Values are given in percent of the nearneighbor distance in the perfect lattice. A positive value indicates outward relaxation.

\begin{tabular}{|c|c|c|c|c|c|c|c|c|c|c|c|c|c|c|}
\hline \multicolumn{3}{|c|}{ Neighbor } & \multicolumn{3}{|c|}{$(+)$} & \multicolumn{3}{|c|}{$(-)$} & \multirow{2}{*}{\multicolumn{3}{|c|}{$\xi_{y}{ }^{(+)}$}} & \multicolumn{3}{|c|}{$(-)$} \\
\hline$L_{1}$ & $L_{2}$ & $L_{3}$ & $\xi_{x}$ & $\xi_{y}$ & $\xi_{z}$ & $\xi_{x}$ & $\xi_{y}$ & $\xi_{z}$ & & & & $\xi_{x}$ & $\xi_{y}$ & $\xi_{z}$ \\
\hline & & & \multicolumn{6}{|c|}{$\mathrm{LiF}$} & \multicolumn{6}{|c|}{$\mathrm{LiCl}$} \\
\hline 1 & 0 & 0 & 12.356 & 0.0 & 0.0 & 16.057 & 0.0 & 0.0 & 7.221 & 0.0 & 0.0 & 14.262 & 0.0 & 0.0 \\
\hline 1 & 1 & 0 & -1.635 & -1.635 & 0.0 & -1.316 & -1.316 & 0.0 & -0.925 & -0.925 & 0.0 & -1.319 & -1.319 & 0.0 \\
\hline 1 & 1 & 1 & 0.160 & 0.160 & 0.160 & 0.186 & 0.186 & 0.186 & 0.324 & 0.324 & 0.324 & 0.248 & 0.248 & 0.248 \\
\hline 2 & 0 & 0 & -0.622 & 0.0 & 0.0 & 1.428 & 0.0 & 0.0 & -2.522 & 0.0 & 0.0 & 3.271 & 0.0 & 0.0 \\
\hline 1 & 2 & 0 & 1.752 & 1.654 & 0.0 & 2.227 & 2.355 & 0.0 & 1.366 & 0.967 & 0.0 & 1.387 & 1.516 & 0.0 \\
\hline 1 & 1 & 2 & -0.567 & -0.567 & -0.455 & -0.432 & -0.432 & -0.432 & -0.282 & -0.282 & -0.155 & -0.412 & -0.412 & -0.348 \\
\hline 2 & 2 & 0 & -0.377 & -0.377 & 0.0 & 0.024 & 0.024 & 0.0 & -0.130 & -0.130 & 0.0 & 0.015 & 0.015 & 0.0 \\
\hline 2 & 2 & 1 & 0.345 & 0.345 & 0.444 & 0.561 & 0.561 & 0.609 & 0.303 & 0.303 & 0.386 & 0.301 & 0.301 & 0.285 \\
\hline 3 & 0 & 0 & 0.589 & 0.0 & 0.0 & 0.454 & 0.0 & 0.0 & -0.387 & 0.0 & 0.0 & 2. 639 & 0.0 & 0.0 \\
\hline \multirow[t]{2}{*}{2} & 2 & 2 & -0.316 & -0.316 & -0.316 & -0.124 & -0.124 & -0.124 & -0.100 & -0.100 & -0.100 & -0.182 & -0.182 & -0.182 \\
\hline & & & \multicolumn{6}{|c|}{$\mathrm{LiBr}$} & \multicolumn{6}{|c|}{ LiI } \\
\hline 1 & 0 & 0 & 5.433 & 0.0 & 0.0 & 13. 839 & 0.0 & 0.0 & 3.444 & 0.0 & 0.0 & 14.108 & 0.0 & 0.0 \\
\hline 1 & 1 & 0 & -0.798 & -0.798 & 0.0 & -1.274 & -1.274 & 0.0 & -0.226 & -0.226 & 0.0 & -1.222 & -1.222 & 0.0 \\
\hline 1 & 1 & 1 & 0.331 & 0.331 & 0.331 & 0.263 & 0.263 & 0.263 & 0.303 & 0.303 & 0.303 & 0.192 & 0.192 & 0.192 \\
\hline 2 & 0 & 0 & -3.134 & 0.0 & 0.0 & 3.861 & 0.0 & 0.0 & -5.370 & 0.0 & 0.0 & 4.427 & 0.0 & 0.0 \\
\hline 1 & 2 & 0 & 1.225 & 0.779 & 0.0 & 1. 232 & 1.359 & 0.0 & 1.188 & 0.784 & 0.0 & 1.097 & 1.019 & 0.0 \\
\hline 1 & 1 & 2 & -0.243 & -0.243 & -0.129 & -0.385 & -0.385 & -0.321 & -0.114 & -0.114 & 0.038 & -0.366 & -0.366 & -0.298 \\
\hline 2 & 2 & 0 & -0.112 & -0.112 & 0.0 & 0.045 & 0.045 & 0.0 & -0.110 & -0.110 & 0.0 & 0.063 & 0.063 & 0.0 \\
\hline 2 & 2 & 1 & 0.268 & 0.268 & 0.352 & 0.264 & 0.264 & 0.236 & 0.277 & 0.277 & 0.366 & 0.178 & 0.178 & 0.171 \\
\hline 3 & 0 & 0 & -0.767 & 0.0 & 0.0 & 3.028 & 0.0 & 0.0 & -1.783 & 0.0 & 0.0 & 4.341 & 0.0 & 0.0 \\
\hline \multirow[t]{2}{*}{2} & 2 & 2 & -0.078 & -0.078 & -0.078 & -0.170 & -0.170 & -0.170 & -0.024 & -0.024 & -0.024 & -0.177 & -0.177 & -0.177 \\
\hline & & & & & \multicolumn{2}{|c|}{$\mathrm{NaF}$} & . & & \multicolumn{6}{|c|}{$\mathrm{NaCl}$} \\
\hline 1 & 0 & 0 & 11.128 & 0.0 & 0.0 & 13.202 & 0.0 & 0.0 & 7.625 & 0.0 & 0.0 & 12.527 & 0.0 & 0.0 \\
\hline 1 & 1 & 0 & -1.965 & -1.965 & 0.0 & -1.465 & -1.465 & 0.0 & -1.517 & -1.517 & 0.0 & -1.367 & -1.367 & 0.0 \\
\hline 1 & 1 & 1 & 0.441 & 0.441 & 0.441 & 0.576 & 0.576 & 0.576 & 0.429 & 0.429 & 0.429 & 0.512 & 0.512 & 0.512 \\
\hline 2 & 0 & 0 & 1. 012 & 0.0 & 0.0 & 1.650 & 0.0 & 0.0 & -0.301 & 0.0 & 0.0 & 3.016 & 0.0 & 0.0 \\
\hline 1 & 2 & 0 & 1. 191 & 1.286 & 0.0 & 1. 620 & 1.805 & 0.0 & 1.052 & 0.802 & 0.0 & 1.232 & 1.467 & 0.0 \\
\hline 1 & 1 & 2 & -0.600 & -0.600 & -0.677 & -0.415 & -0.415 & -0.369 & -0.422 & -0.422 & -0.454 & -0.376 & -0.376 & -0.330 \\
\hline 2 & 2 & 0 & -0.415 & -0.415 & 0.0 & -0.050 & -0.050 & 0.0 & -0.230 & -0.230 & 0.0 & -0.010 & -0.010 & 0.0 \\
\hline 2 & 2 & 1 & 0.305 & 0.305 & 0.277 & 0.523 & 0.523 & 0.443 & 0.249 & 0.249 & 0.267 & 0.374 & 0.374 & 0.293 \\
\hline 3 & 0 & 0 & 1. 435 & 0.0 & 0.0 & 0.776 & 0.0 & 0.0 & 0.587 & 0.0 & 0.0 & 1.820 & 0.0 & 0.0 \\
\hline 2 & 2 & 2 & -0.347 & -0.347 & -0.347 & -0.145 & -0.145 & -0.145 & -0.200 & -0.200 & -0.200 & -0.150 & -0.150 & -0.150 \\
\hline
\end{tabular}


TABLE II (continued).

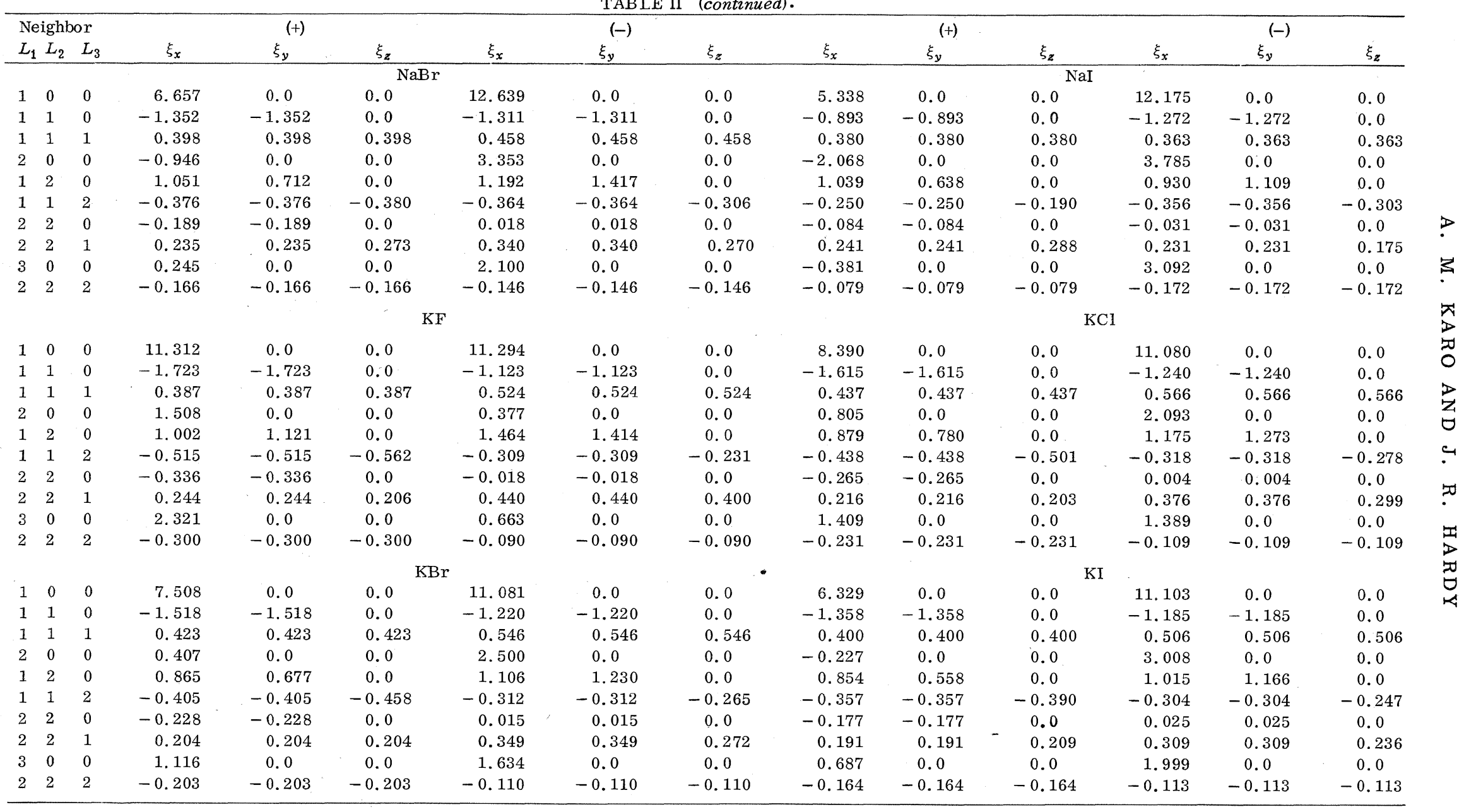


TABLE II (continued).

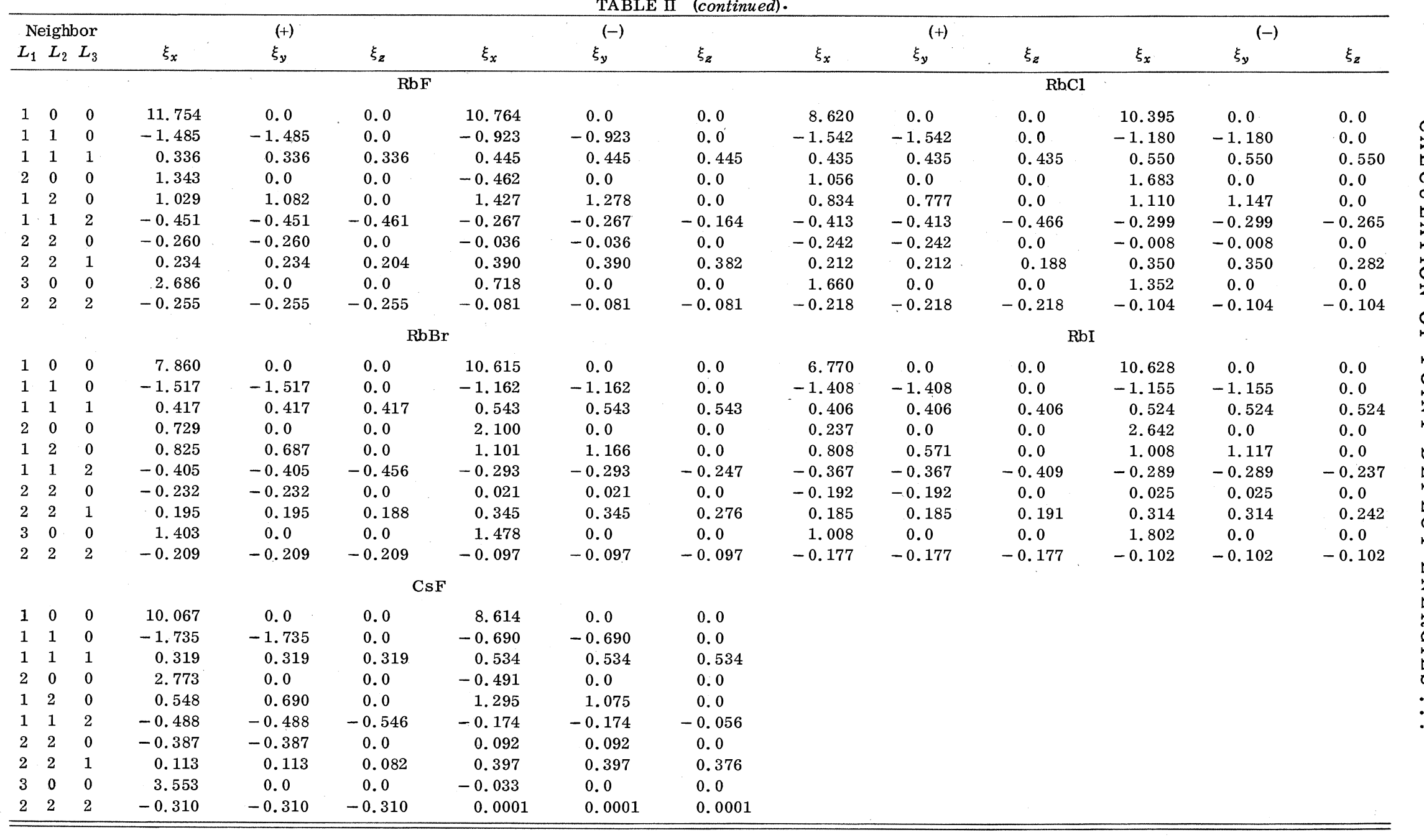


propriate Fourier amplitudes for all the allowed wave vectors after taking into account the effect of first-neighbor relaxation on the short-range force.

The summation of the Fourier series requires additional comment. Both the Fourier series for the displacement and the summation that determines the relaxation energy have integrable singularities in the long-wavelength limit. Throughout the calculations, we have used periodic boundary conditions imposed across the faces of a supercell having the same symmetry as the primitive unit cell of the lattice. To see how the displacements and energies change with the sampling density and to determine whether the singularities at the Brillouinzone origin have introduced complications, we have successively increased the number of points sampled on a uniform mesh within the first Brillouin zone from 1000 to 8000 to 64000 and, in several cases, to 512000 . From this it is found that the displacements converge rapidly for neighbors near the defect. Even for more distant neighbors such as those listed in Table III, the change in going from 8000 to 64000 is only a few percent. Results in Tables II and III for the displacements are for the sampling density of 64000 within the first zone. Increasing the sampling density should not give appreciably different results. There are larger percentage changes in the relaxation energies calculated for sample densities of 8000 and 64000 , respectively, but the convergence is very uniform. Accurate extrapolation to the energy values corresponding to an infinitely dense sample of wave vectors is readily possible. These extrapolated values are those listed in Table I.

In Table I we also have the rcsults obtained by Boswarva and Lidiard ${ }^{10}$ using a refined Mott-Littleton theory. Although very close agreement exists between our results and theirs, it would be unwise to read any great significance into this since the approximations in the two cases are very different. For example, Boswarva and Lidiard used a more highly developed model for the lattice energy, including repulsive interaction beyond first neighbors and also including van der Waals attractions between

TABLE III. $x$ components of the relaxations at lattice sites along three symmetry directions in the lattice. Values are given in terms of percent of the near-neighbor distance in the perfect lattice and positive values indicate outward displacements. The sign in the parentheses indicates the vacancy type. The [100] direction is taken to be equivalent to the $X$ direction.

\begin{tabular}{|c|c|c|c|c|c|c|c|c|c|c|}
\hline \multicolumn{3}{|c|}{ Neighbor } & \multicolumn{2}{|c|}{$\mathrm{LiF}$} & \multicolumn{2}{|c|}{$\mathrm{NaCl}$} & \multicolumn{2}{|c|}{$\mathrm{KBr}$} & \multicolumn{2}{|c|}{$\mathrm{RbI}$} \\
\hline$L$ & $L_{2}$ & $L_{3}$ & $\xi_{x}(+)$ & $\xi_{x}(-)$ & $\xi_{x}(+)$ & $\xi_{x}(-)$ & $\xi_{x}(+)$ & $\xi_{x}(--)$ & $\xi_{x}(+)$ & $\xi_{x}(-)$ \\
\hline 1 & 0 & 0 & 12.356 & 16.057 & 7.625 & 12.527 & 7.508 & 11.081 & 6.770 & 10.628 \\
\hline 2 & 0 & 0 & -0.622 & 1.428 & -0.301 & 3.016 & 0.407 & 2.500 & 0.237 & 2.642 \\
\hline 3 & 0 & 0 & 0.589 & 0.454 & 0.587 & 1.820 & 1.116 & 1.634 & 1.008 & 1.802 \\
\hline 4 & 0 & 0 & -0.273 & -0.280 & -0.220 & 0.462 & 0.055 & 0.415 & 0.032 & 0.539 \\
\hline 5 & 0 & 0 & 0.244 & 0.240 & 0.155 & 0.539 & 0.316 & 0.479 & 0.282 & 0.540 \\
\hline 6 & 0 & 0 & -0.125 & -0.127 & -0.117 & 0.139 & -0.007 & 0.116 & -0.016 & 0.167 \\
\hline 7 & 0 & 0 & 0.132 & 0.127 & 0.075 & 0.256 & 0.142 & 0.220 & 0.124 & 0.244 \\
\hline 8 & 0 & 0 & -0.073 & -0.077 & -0.070 & 0.067 & -0.012 & 0.050 & -0.017 & 0.075 \\
\hline 9 & 0 & 0 & 0.081 & 0.076 & 0.043 & 0.150 & 0.081 & 0.126 & 0.069 & 0.138 \\
\hline 10 & 0 & 0 & -0.047 & -0.051 & -0.046 & 0.040 & -0.009 & 0.028 & -0.013 & 0.043 \\
\hline 1 & 1 & 0 & -1.635 & -1.316 & -1.517 & -1.367 & -1.518 & -1.220 & -1.408 & -1.155 \\
\hline 2 & 2 & 0 & -0.377 & 0.024 & -0.230 & -0.010 & -0.228 & 0.015 & -0.192 & 0.025 \\
\hline 3 & 3 & 0 & -0.136 & 0.043 & -0.080 & -0.021 & -0.095 & -0.001 & -0.084 & -0.006 \\
\hline 4 & 4 & 0 & -0.076 & 0.023 & -0.051 & -0.013 & -0.058 & -0.004 & -0.052 & -0.005 \\
\hline 5 & 5 & 0 & -0.051 & 0.011 & -0.037 & -0.012 & -0.041 & -0.007 & -0.037 & -0.007 \\
\hline 6 & 6 & 0 & -0.036 & 0.006 & -0.028 & -0.010 & -0.030 & -0.007 & -0.027 & -0.007 \\
\hline 7 & 7 & 0 & -0.026 & 0.004 & -0.021 & -0.008 & -0.022 & -0.006 & -0.020 & -0.006 \\
\hline 8 & 8 & 0 & -0.020 & 0.003 & -0.016 & -0.007 & -0.017 & -0.005 & -0.016 & -0.005 \\
\hline 9 & 9 & 0 & -0.015 & 0.002 & -0.013 & -0.005 & -0.013 & -0.004 & -0.012 & -0.004 \\
\hline 10 & 10 & 0 & -0.012 & 0.002 & -0.010 & -0.004 & -0.011 & -0.003 & -0.010 & -0.003 \\
\hline 1 & 1 & 1 & 0.160 & 0.186 & 0.429 & 0.512 & 0.423 & 0.546 & 0.406 & 0.524 \\
\hline 2 & 2 & 2 & -0.316 & -0.124 & -0.200 & -0.150 & -0.203 & -0.110 & -0.177 & -0.102 \\
\hline 3 & 3 & 3 & 0.168 & 0.272 & 0.111 & 0.121 & 0.078 & 0.119 & 0.074 & 0.104 \\
\hline 4 & 4 & 4 & -0.055 & 0.009 & -0.040 & -0.035 & -0.048 & -0.024 & -0.042 & -0.025 \\
\hline 5 & 5 & 5 & 0.065 & 0.109 & 0.042 & 0.044 & 0.028 & 0.043 & 0.027 & 0.038 \\
\hline 6 & 6 & 6 & -0.020 & 0.011 & -0.016 & -0.015 & -0.020 & -0.010 & -0.018 & -0.010 \\
\hline 7 & 7 & 7 & 0.033 & 0.056 & 0.021 & 0.021 & 0.014 & 0.021 & 0.013 & 0.018 \\
\hline 8 & 8 & 8 & -0.010 & 0.008 & -0.008 & -0.008 & -0.011 & -0.005 & -0.009 & -0.006 \\
\hline 9 & 9 & 9 & 0.019 & 0.032 & 0.012 & 0.012 & 0.008 & 0.012 & 0.007 & 0.010 \\
\hline 10 & 10 & 10 & -0.005 & 0.005 & -0.004 & -0.005 & -0.006 & -0.003 & -0.005 & -0.003 \\
\hline
\end{tabular}


second neighbors.

\section{SUMMARY}

The present results are very satisfactory and represent the zero-order approximation. Subsequent extension of this initial investigation to more refined lattice potential functions and to forces evaluated at the relaxed positions has been carried out $^{11}$ for Frenkel-pair formation energies where the relaxations are significantly larger. In modifying the zero-order approximation for $E_{R}$ to allow for relaxation of the first neighbors, the calculations become considerably more complex. How ever, these refinements are essential for treating an interstitial positive or negative ion since the relaxations about the defect are so large that zeroorder results have little significance.

The lattice-statics method, then, avoids the difficulty inherent in the Mott-Littleton approach of matching a discrete region $I$ to a continuum region II. The two regions into which the lattice is divided in the lattice-statics treatment describe the space where the defect-lattice forces are corrected for relaxation and the space where the forces are evaluated at the unrelaxed positions. From our present knowledge, restricting the first region to first neighbors of the defect seems to be sufficient. This is readily checked by computing the displacements and dipoles of various close neighbors. For other than first neighbors, these are usually small.

Thus, the method of lattice statics offers a rigorous means of making self-consistent calculations of charged-defect formation energies in ionic crystals. The next stage in such calculations, after correcting for relaxation effects, would be to use a more sophisticated form for the cohesive energy of the perfect lattice, e.g. , to include secondneighbor, short-range, and van der Waals terms as done by Boswarva and Lidiard ${ }^{10}$ in their MottLittleton calculations. The present results are presented in the spirit of Mott and Littleton's original work: that is, to demonstrate the validity of the technique for a simple potential function. To include all the refinements initially would be to confuse and obscure the power and effectiveness of this new technique. In comparison with experiment the present results show the same tendency as the various Mott-Littleton results to be on the low side. Although more refined calculations may prove better in this respect, it should be noted that experimental values of $E_{S}$ are measured indirectly, depending significantly on theoretical analysis of the experimental data, and are taken at several hundred degrees centigrade, whereas the input parameters generally available for the lattice-statics theory are from experimental data at or below room temperature.

\section{ACKNOWLEDGMENT}

We are indebted to I. W. Morrison of the Computation Division of the Lawrence Radiation Laboratory, University of California, for designing and carrying through the computation reported in this investigation.
*Work performed under the auspices of the U. S. Atomic Energy Commission.

${ }^{1}$ N. F. Mott and M. J. Littleton, Trans. Faraday Soc. $\underline{34}, 485$ (1938).

${ }^{2} \mathrm{P}$. O. Löwdin, $A$ Theoretical Investigation into Some Properties of Ionic Crystals (Almqvist and Wiksells boktryckeri AB, Uppsala, Sweden, 1948).

${ }^{3}$ J. W. Flocken and J. R. Hardy, Phys. Rev. 175, 919 (1968); 177, 1054 (1969).

${ }^{4}$ A. H. Scholz, AERE Report No. R5449; Phys. Status Solidi 25, 285 (1968).

${ }^{5}$ J. R. Hardy and A. B. Lidiard, Phil. Mag. 15, 825 (1967).
${ }^{6}$ J. R. Hardy, Phil. Mag. 7, 315 (1962).

${ }^{7}$ B. Szigeti, Proc. Roy. Soc. (London) A204, 51 (1950).

${ }^{8}$ P. P. Ewald, Ann. Physik 54, 519 (1917); 54, 557 (1917); 64, 253 (1921).

${ }^{9}$ A. M. Karo and J. R. Hardy, Lawrence Radiation Laboratory Report No. UCRL-71475 (unpublished) . This report also contains detailed results pertaining to both the temperature and the model dependence of the lattice-statics method.

${ }^{10}$ I. M. Boswarva and A. B. Lidiard, Phil. Mag. 16, 805 (1967).

${ }^{11}$ P. Schulze and J. R. Hardy, Bull. Am. Phys. Soc. 15, 51 (1970). 